\title{
Nouvelles perspectives en histoire sociale des transports par rail?
}

The social history of railway transportation revisited?

\section{Christian Chevandier}

\section{OpenEdition}

\section{Journals}

Édition électronique

URL : https://journals.openedition.org/rhcf/910

DOI : $10.4000 /$ rhcf. 910

\section{Éditeur}

Rails \& histoire

Édition imprimée

Date de publication : 1 juin 2008

Pagination : 197-209

ISSN : 0996-9403

Référence électronique

Christian Chevandier, "Nouvelles perspectives en histoire sociale des transports par rail ? ", Revue d'histoire des chemins de fer [En ligne], 39 | 2008, mis en ligne le 01 juin 2011, consulté le 22 avril 2022 URL : http://journals.openedition.org/rhcf/910 ; DOI : https://doi.org/10.4000/rhcf.910 
Christian CHEVANDIER

\section{Nouvelles perspectives en histoire sociale des transports par rail ?}

L.

réflexion sur ce qui est souhaitable et possible dans un champ de l'histoire est, comme toute approche épistémologique ou programmatique, inscrite en son temps. Au lecteur des décennies prochaines de faire la part des choses, mais ce texte tente néanmoins de prendre en compte le temps long des questionnements de l'historien confronté à la société cheminote. Et ce temps long ne peut que partir de ce qui a été fait. Et beaucoup a été fait depuis deux décennies dans le cadre de l'AHICF. Cette rapide approche où l'on pourra déceler quelques suggestions commence donc par un inventaire succinct avant de soulever quelques questions, qui peuvent être investies comme autant de champs, et de suggérer quelques méthodes.

\section{Inventaire}

Les Cheminots: ce petit ouvrage de Georges Ribeill a été le livre de chevet de tous les chercheurs en sciences sociales, historiens, sociologues, politistes, ethnologues, qui depuis le milieu des années 1980 se sont penchés sur le monde des travailleurs du rail. Dans la partie bibliographique, l'auteur pouvait écrire : «Les travaux proprement sociologiques consacrés au personnel de la SNCF 
sont plutôt limités ${ }^{1}$. "S'il était alors possible de citer quelques chapitres ou passages d'ouvrages de grands noms de la sociologie, Pierre Belleville et Renaud Sainsaulieu ${ }^{2}$, ainsi que la thèse de J. Curie consacrée à l'analyse du comportement des cheminots d'origine agricole embauchés au début des années soixante en région toulousaine, les textes d'historiens étaient plus rares. À l'exception de la thèse d'Yves Lequin, qui intégrait dans des analyses plus large une approche ponctuelle de certaines populations cheminotes (par exemple les cheminots mariés à Oullins entre 1902 et 1911) ${ }^{3}$, ils renvoyaient avant tout aux luttes sociales et au syndicalisme : la thèse d'Élie Fruit sur les débuts du syndicalisme cheminot en France et, dans le Mouvement social, un article de François Caron sur les mécaniciens de la Compagnies du Nord avant la Grande Guerre. Quant à l'histoire du syndicalisme et des mouvements sociaux, elle avait donné lieu à deux ouvrages, celui de Guy Chaumel au moment de la scission d'aprèsguerre ${ }^{5}$ et près de vingt ans plus tard celui de la Fédération CGT ${ }^{6}$, d'une telle qualité qu'il été largement utilisé par les chercheurs et en grande partie repris pour une autre autobiographie de la fédération après deux décennies ${ }^{7}$. Quant aux travaux les plus approfondis sur le monde cheminot, nous les devons à Georges Ribeill et ils n'existent que sous la forme de dactylogramme ${ }^{8}$, quand bien même ils sont devenus classiques. Le GRECO Travail et travailleurs du Transport permettait jusqu’à la fin des années 1970 à quelques chercheurs de

1- Georges Ribeill, Les Cheminots, Paris, La Découverte, p. 124.

2- Renaud Sainsaulieu, L'Identité au travail, Paris, Presses de la Fondation nationale des sciences politiques, 1985 (première édition : 1977 ; la seconde est revue et complétée).

3- Les Ouvriers de la région byonnaise (1848-1914), t. 1, La Formation de la classe ouvrière régionale, Lyon, Presses universitaires de Lyon, 1974, carte $\mathrm{n}^{\circ} 70$. Sur le syndicalisme des cheminots, voir le t. 2, Les Intérêts de classe et la République, p. 327-335.

4- Élie Fruit, Les Syndicats dans les chemins de fer en France (1890-1910), Paris, Les éditions ouvrières, 1976, 216 pages ; François Caron, « Essai d'analyse historique d'une psychologie du travail. Les mécaniciens et chauffeurs de locomotives du réseau du Nord de 1850 à 1910 ", Le Mouvement social, nº 50 (1965), p. 3-39 (suivi d'une discussion, p. 39-40).

5- Guy Chaumel, Histoire des cheminots et de leurs syndicats, Paris, Marcel Rivière, 1948.

6- Joseph Jacquet (dir.), Les Cheminots dans l'bistoire sociale de la France, Paris, Éditions sociales, 1967.

7- Jean Gacon (dir.), Batailles du rail, Paris, Messidor, 1986.

8- Georges Ribeill, Le Personnel des compagnies de chemins de fer, matériau pour une contribution à la sociologie historique des professions, tome 1, Des origines à 1914, Paris, Développement et aménagement, 1980 ; Le Personnel des compagnies de chemins de fer, t. 2, Les Cheminots en guerre, 1914-1920. Les métamorphoses d'une corporation, Paris, CERTES-ENPC, 1988 ; Le Personnel de la SNCF (19371981). Contraintes économiques, issues techniques, mutations professionnelles et évolutions sociales. Les cours successifs d'une entreprise publique, Paris, Développement et aménagement, 1982. 
diverses disciplines de se rencontrer, dans un cadre plus large que celui qui était limité aux seuls chemins de fer. Nombre d'entre eux ont eu, ensuite, l'occasion de se retrouver, notamment à l'occasion de manifestations de l'AHICF'.

Patrick Fridenson a souligné en 1987 que «l'histoire contemporaine en France a longtemps dédaigné les problèmes de l'organisation du travail. Pour l'essentiel, le travail appartenait aux sociologues et psychologues. Dans la première série des Annales, avant la guerre, le seul article sur le sujet émane ainsi de Georges Friedmann et date de $1935 »^{{ }^{10}}$. Parmi les premiers travaux qui ont marqué la prise en compte de ces problèmes, signalons un article de Georges Ribeill sur les premières années des compagnies ferroviaires ${ }^{11}$. La question a ensuite irrigué l'histoire des chemins de fer et est présente tout au long de l'Histoire des chemins de fer en France de François Caron ${ }^{12}$.

Au début du XXI e siècle, une bibliographie d'histoire sociale des cheminots serait fournie et prendrait largement en compte les travaux menés au sein de l'AHICF, particulièrement sa commission d'histoire sociale. Dès le premier colloque, tenu en mai 1988, une demi-journée était consacrée à «la société cheminote $»^{13}$. Quant au syndicalisme et aux conflits sociaux, deux numéros de la Revue d'histoire des chemins de fer ont été consacrés aux actes de journées scientifiques qui se sont tenues à leur propos. La première ${ }^{14} \mathrm{~s}^{\prime} e s t$ déroulée avant même la création de la commission d'histoire sociale en 1995 et la seconde ${ }^{15}$, près d'une décennie plus tard, correspond à une séance tenue au Centre d'histoire sociale du XX $\mathrm{X}^{\mathrm{e}}$ siècle de l'université Paris 1, rue Malher, qui

9- Par exemple Philippe Corcuff qui a soutenu sa thèse en 1991, «Constructions du mouvement ouvrier. Activités cognitives, pratiques unificatrices et conflits dans un syndicat de cheminots ", École des hautes études en sciences sociales, et Atsushi Fukasawa dont la thèse, "Histoire du syndicalisme cheminot en France. Des grèves générales à la Grande Guerre », préparée à l'université de Paris 1 sous la direction d'Antoine Prost fut soutenue en décembre 1992.

10- Patrick Fridenson, «Un tournant taylorien de la société française (1904-1918) », Annales Économies Sociétés Civilisations, $\mathrm{n}^{\circ}$ 42/5 (septembre-octobre 1987), p. 1031-1060.

11- Georges Ribeill, «Gestion et organisation du travail dans les Compagnies de chemin de fer des origines à 1860 ", Annales Économies Sociétés Civilisations, n $42 / 5$ (septembre-octobre 1987), p. 999-1029.

12- François Caron, Histoire des chemins de fer en France, tome premier, 1740-1883, et tome second, 1883-1937, Paris, Fayard, 1997 et 2005.

13- Le titre même du colloque introduisait une histoire sociale qui ne serait pas limitée au monde des cheminots, «Les Chemins de fer, l'espace et la société en France », RHCF HS 1 [Les références des publications de l'AHICF sont développées dans la bibliographie, p. 325 ].

14- RHCF 3.

15- RHCF 19. 
avait été quelque peu perturbée par une grève de la SNCF. Là ne s'est d'ailleurs pas arrêtée l'œuvre de la commission puisque mon ouvrage sur les Cheminots en grève a été en bonne partie le fruit de travaux ou de discussions menés dans le cadre de la commission, notamment l'étude de la grève de juin $1971^{16}$. Quant au Maitron des cheminots ${ }^{17}$, avant qu'il soit pris en charge par le Comité central d'entreprise de la SNCF, son élaboration avait été entreprise dans le cadre de la commission. Tous ces travaux ont été très féconds et ont permis qu'une histoire scientifique existe largement, pas toujours sans difficulté, à côté d'une histoire militante elle-même renforcée par la création en 1998 de l'Institut d'histoire sociale de la Fédération CGT des cheminots.

Parmi les travaux de la commission d'histoire sociale de l'Association pour l'histoire des chemins de fer en France, soulignons la recherche coordonnée de démographie historique qui a permis de donner sa juste place à la légende d'une hérédité professionnelle des cheminots et d'un groupe social qui se reproduirait lui-même. Associant des généalogistes, des étudiants en deuxième ou troisième cycle et des chercheurs confirmés, elle s'est en outre déroulée selon des modalités, notamment de respect mutuel, qui correspondent à ce que l'on est en droit d'attendre d'une société scientifique ${ }^{18}$. Enfin, l'AHICF et le Centre d'histoire sociale du XXe siècle (université de Paris 1/ CNRS) ont organisé de 2002 à 2005, avec le Service du livre du Comité central d'entreprise de la SNCF, le séminaire « Les cheminots, images et représentations croisées » puis, en mars 2006, avec le Centre des archives du monde du travail, le colloque «Images de cheminots. Entre représentations et identités ». Les actes en ont été publiés en un seul volume ${ }^{19}$.

Une des spécificités de la recherche menée au sein de l'AHICF est ce rapport qu'entretiennent en son sein les chercheurs tout comme les travailleurs des chemins de fer, à quelque niveau de la hiérarchie qu'ils se trouvent et quels que soient leurs spécialisations et leurs engagements. Ces rapports ne peuvent être qu'ambigus, tant il est peu plaisant d'être objectivé et parce que la présence

16- Deux séances, les 26 avril et 21 juin 2001, se sont tenues à ce propos au Centre d'histoire sociale du XXe siècle. Marie-Louise Goergen, Michel Gorand, Georges Ribeill, Pierre Vincent et moi-même avons étudié à cette occasion les archives syndicales et de police ainsi que celles de la SNCF, dépouillées par Georges Ribeill.

17- Marie-Louis Goergen, Cheminots et militants. Un siècle de syndicalisme ferroviaire, Paris, Éditions de l'Atelier, 2003.

18- « Origines sociales et géographiques des cheminots français », RHCF 22.

19- «Images de cheminots, entre représentations et identités », RHCF 36-37. 
même de professionnels du rail dans une telle association souligne l'intensité de leur identité professionnelle. «Ce que demandent les groupes mémoriels, quels qu'ils soient, aux historiens, est moins le résultat de leurs recherches, par définition toujours ambivalentes, toujours irrécupérables, que des arguments en faveur de leur juste cause. Il revient donc aux historiens de trouver les voies d'un dialogue difficile ${ }^{20}$. » Il n'est pas pour autant facile à beaucoup de cheminots de lire que la principale faiblesse des mouvements sociaux dans les chemins de fer tient dans la division : clivage syndical d'abord, dispersion entre réseaux et régions, avec des tendances qui se repèrent sur un temps très long, segmentation entre les différents groupes professionnels au sein de la corporation, les mécaniciens se substituant dans les années 1950 aux ouvriers d'atelier comme le groupe le plus vindicatif. Ce serait pécher par naïveté que de croire que le constat de l'éclatement du groupe social pourrait être innocemment posé. Remarquons par ailleurs que l'intérêt bien compris des institutions dérangées par les questions de l'historien devrait passer par la réponse à ces questions. Celle des divisions, catégorielles, régionales, d'organisation, revient de manière récurrente dans le syndicalisme cheminot et c'est bien du refus de se confronter à cette réalité, quand ce sont des militants autonomes qui conduisent les trains lors des grèves de la CGT ou des militants cégétistes qui conduisent les trains lors des grèves du syndicat autonome, que provient la vulnérabilité du mouvement social des travailleurs des chemins de fer.

\section{Questions}

Précisons d'abord que les sujets déjà étudiés n’ont pas été épuisés. Ainsi, les nombreux travaux sur les grèves, les grévistes, les syndicats et les militants rendent d'autant plus nécessaire une histoire de ceux qui se sont opposés aux grèves ou, plus simplement, se sont abstenu de tout engagement dans un milieu syndical qui était, dans les chemins de fer, profondément réformiste. Il y aurait bien sûr les " jaunes ", les " renards » stigmatisés lors des grandes grèves, mais aussi ceux qui, en faisant acte de présence à leur poste lors d'une grève, se tenaient à l'écart de leurs compagnons de travail et ont été qualifiés lors d'une assemblée générale du mouvement de novembre 2007 comme « les copains et copines qui ne sont pas en grève aujourd'hui $»^{21}$. Le comportement

20- «Ils n’y parviennent pas toujours et se contentent de peu » conclut Christophe Prochasson, L'Empire des émotions. Les historiens dans la mêlée, Paris, Demopolis, 2008, p. 210.

21- Assemblée générale des grévistes du dépôt du Charolais, 14 novembre 2007 ; mis en perspective, de tels propos signalent une singulière évolution. 
de ces « passagers clandestins » (free riders ${ }^{22}$ ), qui profitent des résultats de l'action des autres sans s'impliquer directement, gagnerait ainsi à être étudié, au-delà même de ce qu'apporte le paradigme utilitariste, et nous aurions là un observatoire pour mieux comprendre la société cheminote, parce que, comme le cheminot gréviste, celui qui s'abstient s'inscrit volens nolens dans un corpus plus large. Que ce soit en dépit ou du fait même de cette identité sociale profondément réformiste ${ }^{23}$, l'importance des mouvements sociaux dans les chemins de fer est telle que nous ne pouvons laisser cet aspect de côté. Il n'est pas paradoxal de penser que le conflit social participe de l'investissement des agents et donc de la qualité, en France, de l'activité ferroviaire. Il faut alors se remettre à l'ouvrage, approfondir et comprendre en quoi les a-grévistes, les non-grévistes, les opposants à la grève contribuent à construire le caractère et la nature d'un mouvement social.

L'impressionnant bilan ne rend pas superflue toute nouvelle recherche, mais celle-ci devrait précisément s'appuyer sur ce qui a été fait, non pour simplement élargir ou préciser, mais parce que de nouvelles problématiques peuvent questionner les mêmes faits ou parce qu'il est possible de les appréhender sur un temps plus long, qui reformule alors nos questionnements. Lorsque, le 12 décembre 1995, Pierre Bourdieu a animé, gare de Lyon, une rencontre entre les grévistes et des intellectuels, il était tentant de mettre en perspective cette réunion et la manifestation du 24 mai 1968, lorsque des étudiants qui manifestaient dans le quartier et qui étaient poursuivis par les forces de l'ordre n'ont pu se réfugier dans la gare de Lyon bouclée par la CGT $^{24}$. Mais l'évolution ne s'arrête pas là : le 30 mars 2006, dans le cadre du mouvement contre le Contrat première embauche, lorsque des centaines d'étudiants occupèrent les voies de la gare de Lyon, ce sont des cheminots qui permirent à la plupart d'entre eux d'échapper, cette fois, à la police ${ }^{25}$.

22- Mançur Olson, La Logique de l'action collective, Paris, PUF, 1978.

23- Le fait que les attaques contre le groupe social des cheminots sont considérées comme emblématiques, dans le cadre de la dérégulation de l'économie et des contre-réformes qui défont l'État social, devrait d'ailleurs amener à se demander si ce n'est pas précisément l'identité réformiste du groupe social qui est à l'origine des mouvements sociaux qui ont marqué les chemins de fer français depuis le milieu des années 1980 et qui ont tous eu un caractère défensif. Seule exception, le mouvement de 2003 a cessé lorsque le ministre et le président de la SNCF ont promis aux agents de la SNCF qu'ils conserveraient leur régime spécial de retraite, "Chapitre 4, Les régimes particuliers des services publics », Michel Pigenet (dir.), Retraites. Une histoire des régimes spéciaux, Paris, ESF Editeur, 2008, p. 53-70, notamment p. 67 la déclaration du ministre des Transports, Gilles de Robien.

24- Christian Chevandier, Cheminots en grève, ou la construction d'une identité (1848-2001), Paris, Maisonneuve et Larose, 2002, p. 299.

25- Loin des censiers battus. Témoignages et documents sur le mouvement contre le CPE et la précarité, Paris, Éditions CNT, 2007, p. 131. 
Les travaux sur la mobilité sociale et géographique des cheminots français, complétés et confirmés ${ }^{26}$ depuis la publication de la recherche menée dans le cadre de la commission d'histoire sociale des transports par fer, qui font qu'il s'agit aujourd'hui sans doute de la population la mieux connue à cet égard, peuvent être poussés plus avant. Si ces études ont, en dépit de quelques incursions jusqu'au milieu du XX' siècle, surtout porté sur le temps d'avant la Grande Guerre, il faudrait sans doute établir une périodisation de l'hérédité cheminote, voir comment elle a pu être un remède ou un antidote aux crises génératrices de chômage, pouvoir saisir le devenir des enfants de ce groupe malthusien. Il conviendrait de savoir aussi ce que deviennent les cheminots lorsqu'ils quittent les chemins de fer. Si une histoire des retraites des cheminots a pu être écrite ${ }^{27}$, il reste à rédiger une histoire des retraités des chemins de fer. Déchargés tôt de la charge du labeur, ils sont alors disponibles et l'on est curieux de voir si ce temps est alors mis, par des engagements associatifs ou édilitaires, à la disposition de la population, ce qui correspondrait à ce caractère ouvert du groupe social mis en évidence à de nombreuses reprises. Les études sur les représentations des cheminots peuvent être complétées par des recherches d'ethnologues ou de sociologues. Nous avons à plusieurs reprises, dans la continuité des travaux de Michel Ionascu ${ }^{28}$, abordé la place des cheminots dans l'art cinématographique et un numéro de CinémAction est consacré en 2009 au «train au cinéma » ${ }^{29}$. Le théâtre cependant n’a pas été véritablement interrogé, alors que des pièces populaires qui mettaient en scène au XIX ${ }^{\mathrm{e}}$ siècle cette nouvelle technologie à une création du début du XXI' siècle, Les Pas

26- David Lamoureux, «Les cheminots du Sud-Est de la France de la démobilisation au Front populaire. Hommes, agents, syndiqués ", thèse d'histoire contemporaine, université de Nice, 2004, notamment le tome 1, «Devenir cheminot».

27- Georges Ribeill, Des faveurs patronales au privilège corporatif. Histoire du régime de retraites des cheminots des origines à nos jours (1850-2003), Dixmont, chez l'auteur, 2003. Voir aussi les Cabiers des Caisses de prévoyance et de retraite, revue générale de Sécurité sociale.

28- Michel Ionascu, «Cheminots argentiques : l'image d'un groupe social dans le cinéma et l'audiovisuel français ", thèse de recherches cinématographiques et audiovisuelles, université de Paris III, 1999. Il a notamment étudié un corpus d'une quarantaine de longs métrages, d'une vingtaine de courts métrages de fiction, de onze téléfilms de fiction, de deux feuilletons télévisés, de dizaines de reportages pour la télévision, de deux documentaires syndicaux, de documentaires "indépendants » ainsi que de fictions tournés par des cheminots cinéastes amateurs, regroupés notamment au sein des clubs de l'Union artistique et intellectuelle des cheminots français.

29- Sous la direction d'Albert Montagne. La revue n'avait pas auparavant ignoré les chemins de fer. Voir ainsi, à propos de deux films largement abordés dans le n 36-37 de la Revue d'bistoire des chemins de fer, Sylvie Dreyfus, "Deux films qui se répondent : Les Rendez-vous de décembre et Nadia et les hippopotames », CinémAction, $\mathrm{n}^{\circ} 110,1^{\text {er }}$ trimestre 2004, p. 186-188. 
perdus $^{30}$, le corpus est loin d'être négligeable. Il est un autre domaine qui devrait être plus largement exploré, celui de la pratique de l'écriture dans le monde des travailleurs du rail. Il est pour le moins surprenant que, de Georges Valero à Maxime Vivas, il soit si facile d'écrire sur les postiers romanciers ou poètes et qu'il se révèle malaisé de dénicher un écrivain cheminot ${ }^{31}$, un véritable travailleur du rail qui ne serait ni Jacques Valdour, ni Pierre Hamp, ni Henri Vincenot. Alors que la « littérature d'expression populaire $»^{32}$ est si riche pour d'autres groupes sociaux tel celui des travailleurs de la mine ${ }^{33}$, des textes de cheminots, notamment des autobiographies, restent à dénicher. À moins qu'il ne faille s'interroger sur l'indigence qu'en révèle le dénombrement.

La période de la Deuxième Guerre mondiale et de l'Occupation a été largement abordée. Deux ouvrages sur la Résistance des cheminots, emblématiques l'un de la mémoire gaulliste ${ }^{34}$ et l'autre de la mémoire communiste ${ }^{35}$, s'étaient répondu à la fin des années 1960. Depuis, deux colloques de l'AHICF en ont traitée ${ }^{36}$ et elle a fait l'objet d'un chapitre d'un ouvrage ${ }^{37}$, mais le sujet est loin d'avoir été épuisé comme l'on montré les communications de Laurence Bour, Georges Ribeill et Marie-Noëlle Polino à un colloque du groupement de recherches du CNRS «Les entreprises françaises sous l'Occupation »" Là

30- Pièce de Denise Bonal mise en scène par Gilles Guillot, Théâtre du Rond-Point, 6 septembre au 29 octobre 2005.

31- Sophie Béroud, Tania Régin (coord.), Le Roman social, littérature, histoire et mouvement ouvrier, Paris, Éditions de l'Atelier, 2002.

32- Michel Ragon, Histoire de la littérature prolétarienne de langue française, Paris, Albin Michel, 1986.

33- Diana Cooper-Richet, Le Peuple de la nuit. Mines et mineurs en France, XIXe-XXe siècles, Paris, Perrin, 2002 ; un chapitre entier développe "l'imaginaire de la mine » et l'auteur souligne (p. 209) la place du succès de Germinal dans le passage à l'acte d'écrire de nombreux mineurs. Cela fut-il le cas pour un autre roman d'Emile Zola, La Bête bumaine, et, si ce ne fut pas le cas, pourquoi?

34- Paul Durand, La SNCF pendant la guerre, sa résistance à l'occupant, Paris, Presses universitaires de France, 1968. Voir auparavant, du même auteur, «La politique de l'emploi à la SNCF pendant la Deuxième Guerre mondiale », Revue d'bistoire de la Deuxième Guerre mondiale, janvier 1965, p. 19-40. 35- Marcel Choury, Les Cheminots dans la bataille du rail, Paris, Librairie Perrin, 1970.

36- Une entreprise publique pendant la guerre : la SNCF, 1939-1945, Paris, Presses universitaires de France, 2001 et "Les cheminots dans la Résistance. Une histoire en évolution », actes de la journée du 3 décembre 2005, RHCF 34.

37- Christian Chevandier, Cheminots en grève, op. cit., chapitre III, « Les batailles du rail (19391944) », p. 155-218.

38- « La réquisition des cheminots pour le travail en Allemagne. L'apport des archives de la SNCF », «Entre effectifs réduits et besoins accrus, quelques aspects de la gestion du personnel de la SNCF » et «La réquisition des cheminots pour le travail en Allemagne. Une étude de cas », in Christian Chevandier et Jean-Claude Daumas (dir.), Travailler dans les entreprises en France sous l'Occupation, Dijon/Besançon, Presses universitaires de Franche Comté, 2007, p. 131-135, 137 153 et $155-174$. 
aussi, comme a été souligné l'intérêt d'une étude des non-grévistes au sein de ce groupe social supposé porté sur la lutte sociale, il serait intéressant de se pencher sur les collaborateurs et les pétainistes d'une corporation parfois présentée comme unanimement résistante. Est-il ainsi vraiment impossible de dénicher le dossier du cheminot Paul Touvier ? Quant aux trains de la déportation, aussi rares furent-ils parmi un trafic développé, répétons qu'il faudrait se pencher sur ceux qui ont pu les conduire, les identifier ${ }^{39}$, recueillir avec précaution leurs témoignages s'il est encore temps. Un autre champ reste à défricher, qui nous rappelle que l'histoire sociale des transports par rail n'est pas uniquement l'histoire sociale des cheminots, c'est celle des voyageurs. Des voyageurs, pas des clients, puisqu'une telle approche nécessiterait de ne pas se contenter d'une simple démarche d'histoire économique ou de la gestion. Les travaux de Nels Anderson sur les bobos ${ }^{40}$ ne pourraient certes pas trouver un équivalent dans notre pays, mais il n'en reste pas moins qu'une étude sur un temps long des pratiques de voyage en fraude serait à mener. Plus largement, une étude de la violence exercée dans les chemins de fer, à l'encontre des usagers comme du personnel, devrait être perçue comme indispensable. Nombre des prochains mouvements sociaux pourraient être appréhendés avec plus de pertinence si nous comprenions pourquoi et depuis quand il est insupportable pour un travailleur des chemins de fer d'être la victime de violences, voire que les voyageurs le soient.

Surtout, une histoire sociale des chemins de fer ne peut être hermétiquement isolée. Elle ne peut se développer sans prendre en compte les apports de l'histoire économique, ceux de l'histoire des techniques, de l'histoire des innovations, et nous savons à quel point cet élément est essentiel. Mais n'oublions pas non plus l'histoire politique. En quoi le chemin de fer contribue-t-il à construire la cité, à construire la nation ? Peu avant sa mort, au colloque de Chateauvallon, Fernand Braudel datait l'unité de la France des débuts du chemin de fer (et aussi de l'école primaire) ${ }^{41}$. En cela, la dimension politique du plan Freycinet est essentielle et la technologie ferroviaire a bien contribué à construire la France en la quadrillant, en reliant les Français, de Dunkerque à Hendaye, de Brest à Menton. La construction politique de l'Europe passera, ainsi, par le TGV, mais peut-être également par l'action sociale des

39- Pour des recherches où serait respecté leur anonymat, l'historien n'ayant pas la vocation de sycophante et l'obtention de dérogations pour l'accès aux dossiers du personnel ou de retraite en étant ainsi facilitée.

40- Nels Anderson, The American Hobo: an Autobiography, Leiden, E. J. Brill, 1975.

41- Les actes en ont été publiés l'année suivante sous le titre: Une Leçon d'histoire, Paris, Arthaud, 1986. 
cheminots européens. La dimension politique du chemin de fer est, sans nul doute, un des facteurs des engagements des cheminots français, que ce soit dans la Résistance ou dans les mouvements sociaux de la fin du XX et du $\mathrm{XXI}^{\mathrm{e}}$ siècle, lorsque le groupe social des travailleurs du rail s'est perçu comme investi d'une mission à l'échelle de l'ensemble du salariat (" À quoi ça sert d'avoir une histoire si on la ramène pas ? ” ${ }^{42}$ ). Là aussi, l'histoire politique ne pourra faire fi des représentations. De longue date, la perception de ce rôle est franche : sur la façade de la préfecture du département du Rhône, édifiée dans les années 1880 face à la basilique de Fourvière, deux médaillons circulaires représentent l'un un métier à tisser, ce qui n'a rien pour nous surprendre en la ville des canuts, mais l'autre une locomotive, évocation certes de la construction de matériel ferroviaire très développée dans l'agglomération lyonnaise $\mathrm{s}^{43}$, mais célébration également dans une ville girondine du moyen de transport qui rapproche de la capitale.

Toutes ces problématiques nécessiteraient, en un jeu d'échelles, une approche comparative. Lors du séminaire "Les cheminots, images et représentations croisées » puis du surtout du colloque «Images de cheminots. Entre représentations et identités », une attention particulière a été apportée aux éléments qui comportent ou permettent une telle démarche, entre le monde des chemins de fer dans les divers pays mais également entre les différents groupes professionnels et sociaux. Les tentatives de mise en rapport qui permettent de confronter pour comprendre doivent s'effectuer à tous les niveaux possibles et ne peuvent donc pas éluder une comparaison entre les différentes compagnies, voire entre les villes. Les travaux sur le personnel des omnibus et tramways lyonnais menés par Florent Montagnon, dont un texte est publié dans ce volume, sont enrichis des résultats d'une étude des travailleurs de transports en commun parisiens ${ }^{44}$, mais permettent aussi d'en préciser les spécificités. C'est, bien sûr, le cas pour l'histoire des conflits sociaux tant il est vrai qu'elle ne peut que s'intégrer dans une histoire plus large des rapports sociaux ${ }^{45}$.

42- Voir, à propos du film Nadia et les hippopotames, le débat entre historiens et cinéaste, http://www.ahicf.com/commissions.htm.

43- Si l'atelier de Marc Seguin, à Perrache, avait fermé dès 1851, les Chantiers de la Buire à la Guillotière, les Ateliers Jules Weitz à la Mouche et bien sûr les Ateliers PLM d'Oullins comptaient parmi les industries les plus florissantes de l'agglomération.

44- Noëlle Gérôme, Michel Margairaz, Métro, dépôts, réseaux. Territoires et personnels des Transports parisiens, Paris, Publications de la Sorbonne, 2002.

45- Patrick Fridenson, «Le conflit social », in André Burguière et Jacques Revel (dir.), Histoire de la France, volume 3, dirigé par Jacques Julliard, L'État et les conflits, Paris, Le Seuil, 1990, p. 351-453. 


\section{Méthodes}

Les travaux des ethnologues et des sociologues, en dépit de la place prise dans ce champ par Georges Ribeill ${ }^{46}$ qui combine les approches de diverses sciences sociales, présentent le risque de fixer chaque chercheur dans son identité disciplinaire. Or, pour les chemins de fer comme pour l'ensemble des activités, c'est un véritable gâchis qu'entraîne ce respect formaliste et frileux. Il n'y a pas à craindre de se lancer dans des démarches qui ne paraissent étrangères que par convention, et que d'aucuns n'ont pas hésité à pratiquer. «Certains historiens ont ainsi pratiqué l'observation participante comme des chercheurs d'autres sciences sociales, tout en gardant le souci de croiser les résultats de cette observation avec d'autres sources. Le chercheur acquiert ainsi avec son objet de recherche une familiarité et une compréhension "éprouvée" qui transparaissent immédiatement à la lecture du travail universitaire, enrichissent le questionnement et les apports historiques et singularisent les travaux relevant de cette démarche ${ }^{47}$. » Une histoire sociale des cheminots peu aussi passer, pour un chercheur, par des voyages dans la cabine d'un conducteur qui n'excluent pas la conduite (très encadrée !) de locomotives, l'assistance pendant des conflits sociaux à des assemblées générales où, sans prendre la parole, le chercheur peut participer à certains rituels, comme celui d'alimenter un feu en allant chercher des morceaux de bois. La qualité des travaux de certains sociologues vient notamment de leur présence ${ }^{48}$; au demeurant, elle permet de mieux comprendre un milieu qu'en se limitant à des sources habituelles. Le risque est bien sûr celui de l'anachronisme, mais à l'historien de prendre garde ! Le risque d'anachronisme passe aussi par la confusion entre différents documents. Nous avons tous en tête la phrase de Jean Gabin à Simone Simon à propos de la Lison: "Quant on aime quelqu'un, on n'a pas idée de l'appeler avec un numéro. » Dans le roman, ce n'est pas un dialogue des deux personnages,

46- Que le lecteur qui serait tenté de voir dans la présente contribution un long panégyrique de Georges Ribeill lise nos travaux respectifs pour constater qu'il arrive parfois à nos approches de ne point converger.

47- Catherine Omnès, «Les historiens et la tentation ethnographique », in Anne-Marie Arborio, Yves Cohen, Pierre Fournier, Nicolas Hatzfeld, Cédric Lomba et Séverin Muller (dir.), Le Travail, l'histoire et l'ethnologie, Paris, La Découverte, collection "Recherches », 2008, p. 281 297. Voir également, dans le même volume, Nicolas Hatzfeld, « Retour en chaîne et histoire d'usine. Une interférence de temporalités », p. 137-152 ; Jean Peneff, « La lecture d'historiens, une double invitation à l'ethnographie », p. 169-184 et Yves Cohen, "Conclusion : présent/ passé, ethnographie/histoire », p. 313-324.

48- Comment, ici, ne pas penser à Didier Leschi, « La construction de la légitimité d'une grève : le rôle des assemblées générales de la gare de Lyon », Sociologie du travail, n 4/1997, p. 425-448? 
mais une explication plus neutre : "Ainsi que les autres machines de la Compagnie de l'Ouest, en dehors du numéro qui la désignait, elle portait le nom d'une gare, celui de Lison, une station du Cotentin. Mais Jacques, par tendresse, en avait fait un nom de femme, la Lison, comme il disait, avec une douceur caressante. ${ }^{49}$ » Lors de son enquête préalable, Zola avait seulement noté : «Les trains et les machines sont désignés par des numéros. Les machines ont des noms, mais pour le pittoresque ${ }^{50}$. » Mais si nous avons la chance, en ce cas, de bien pouvoir distinguer ces différents textes et leurs natures respective, elle est pour le moins inaccoutumée et, somme toute, du moins pour le texte initial ${ }^{51}$, récente.

Il n'en reste pas moins que la fine connaissance du milieu est indispensable. Jacques Julliard, après la relation d'une discussion avec Gilbert Declercq qui lui racontait une grève ( À la fin, chacun de nous, de son côté, était devenu meilleur » expliquait le syndicaliste, parlant des grévistes et des patrons), commente : «C'est une situation à la Sorel, à la Péguy, à la Pascal même. » Plus loin, il écrit : «Ce que j’appelle ici transcendance peut donc effectivement surgir du temporel. Quiconque a participé à une grande expérience collective sait très bien que ce qui s'est passé excède toujours les résultats obtenus ${ }^{52}$. » À la lecture de certains passages de cet ouvrage, comment ne pas penser à ce bel article d'Annie Kriegel, après la grève de l'été 1953, dans lequel elle a voulu «noter quelques images, rassembler quelques traits de ces heures-là, exaltantes et dures $»^{53}$. Identifier explicitement le caractère exceptionnel de ces moments est au demeurant un privilège d'intellectuel, la culture ouvrière incorporant une très forte pudeur de ces émotions-là, sauf peut-être dans des tête-à-tête où l'on se laisse aller mais où le chercheur est rarement présent. Rien donc, ici, de spécifiquement ferroviaire, mais une démarche qui peut se retrouver peu ou prou pour l'ensemble du monde du travail des milieux populaires ${ }^{54}$.

Le grand chantier de recueil de sources orale ${ }^{55}$, précédé notamment par une réflexion méthodologique et épistémologique ${ }^{56}$, se situe dans la continuité

49- La Bête humaine, Paris, Le livre de poche, 1965, p. 273.

50- Carnets d'enquête. Une ethnographie inédite de la France, Paris, Plon, 1986, p. 499-553.

51- Les Carnets d'enquête d'Emile Zola n'ont été publiés qu'en 1986. Rares était les chercheurs qui, tel Henri Mitterand, les avaient consultés à la Bibliothèque nationale.

52- Le Choix de Pascal, Paris, Desclée de Brouwer, 2003, p. 308-309.

53- Annie Besse, "Ce que furent les grèves du mois d'août ", La Nouvelle Critique, n 48 (septembre-octobre 1953), p. 17-34.

54- Richard Hoggart, La Culture du pauvre, Paris, Éditions de Minuit, 1970.

55- Voir notamment la RHCF 31.

56- « De la série à l'individu. Archives du personnel et archives orales », La Gazette des Archives, $n^{\circ} 198(2005 / 2)$. 
des entretiens qui ont été menés par la plupart des chercheurs cités dans ces lignes. Surtout, le cadre de l'histoire des hommes et des femmes au travail y est aisément délimité ; il n'en est pas moins bien difficile à respecter, et c'est ainsi qu'il a bien fallu prendre garde dans ce programme de collecte d'archives orales sur les métiers à la SNCF depuis 1945 à ne pas susciter des récits de vie qui éloignent par trop du labeur dans les chemins de fer. Sans doute ses modalités éviteront-elles des mésaventures, comme la suspension de la publication de l'entretien avec André Beynet effectué à son domicile par Georges Ribeill le 24 octobre 1988. Il avait été, dans un premier temps, envisagé de publier ce texte dans le $\mathrm{n}^{\circ} 19$ de la Revue d'histoire des chemins de fer.

Dernier point, au croisement des méthodes, des champs et, déjà, du bilan. Ne serait-ce que pour critiquer les résultats antérieurs, donc les méthodes, il conviendrait que les chercheurs qui ont travaillé sur le monde des travailleurs du rail mettent pour leurs successeurs leurs archives de côté. Mais si les conversations téléphoniques s'envolent, peut-être l'archivage de sources numériques est-il assez commode pour que les échanges électroniques demeurent, par exemple ceux qui ont été menés lors de la rédaction commune de quelques notices du Dictionnaire de la Résistance et de la Libération" ("Louis Armand »; « Cheminots »; « Sabotages ferroviaires »; « Résistance fer »). Les historiens de demain auront peut être l'usage de ces sources de choix et sans doute exceptionnelles ${ }^{58}$.

Cette tentative pour dégager de nouvelles perspectives peut sembler peu innovante. Le seul fait qu'elle commence par un inventaire accorde toute leur place aux questionnements anciens. Ne nous y trompons pas : même dans le domaine scientifique, il est malaisé de penser l'avenir autrement que dans les termes du présent, c'est-à-dire du passé5 ${ }^{9}$. Les sciences sociales, même l'histoire, ne font que tenter de répondre aux questions contemporaines. Qui aurait ainsi prévu, au moment de la publication de Les Cheminots, en 1984, que tant de recherches historiques, au cours des décennies suivantes, concerneraient les mouvements sociaux et le syndicalisme dans les transports par fer, le terrain qui semblait déjà le mieux balisé ?

57- François Marcot (dir.), Dictionnaire bistorique de la Résistance, Paris, Robert Laffont, 2006. 58- Si des correspondances entre historiens ont été conservées - et publiées -, les conversations ou les entretiens téléphoniques au cours desquels se sont élaborés de tel textes n’ont bien sûr pas laissé de trace.

59-1900. Comment ils voyaient le nowveau siècle ? Comment ils voyaient l'avenir? Colloque de l'université Paris 1, 28-29 octobre 1999, Boulogne-Billancourt. 
